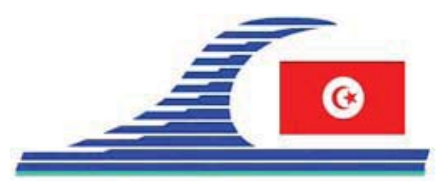

Conférence Méditerranéenne Côtière et Maritime EDITION 1, HAMMAMET, TUNISIE (2009)

Coastal and Maritime Mediterranean Conference

Disponible en ligne - http://www.paralia.fr-Available online

\title{
Etude de l'agitation liée aux vagues dans un bassin portuaire de forme circulaire
}

\author{
Martin SANCHEZ ${ }^{1}$, Damien GRIMONT ${ }^{2}$, Georges CLAVERIE $^{3}$
}

1. Université de Nantes, UMR 6112 CNRS - LPGN, BP 92208, 44322 Nantes, France. martin.sanchez@univ-nantes.fr

2. BLUERING, 5 avenue Cauchy, BP 10703, 44300 Nantes, France. contact@bluering.fr

3. CREOCEAN, Zone Technocean, rue Charles Tellier, 17000 La Rochelle, France. claverie@creocean.fr

\section{Résumé :}

Cette étude est effectuée dans le cadre du projet collaboratif MAREVA (MAquette de REalisation Virtuelle des Aménagements portuaires), porté par SCE Aménagement \& Environnement, labellisé par le Pôle Génie Civil Ecoconstruction et soutenu financièrement par le Fonds Unique Interministériel. Dans cette note sont abordées les contraintes d'agitation liées aux vagues en relation avec le développement du nouveau concept d'aménagement portuaire breveté BlueRing ${ }^{\circledR}$. Dans sa configuration de base, il s'agit d'un port circulaire avec dans sa partie centrale un puits d'accès à un espace souterrain aménagé. Sa configuration en symétrie de révolution, semblable à celle du cothon de Carthage, permet l'obtention d'un linéaire de quais utiles maximum.

\section{Mots-clés :}

Port de plaisance - Port à flot - Port à sec - Houle - Clapot - Agitation - Helmholtz Travaux maritimes - Paroi moulée - BlueRing ${ }^{\circledR}$

\section{Introduction}

Cette étude concerne un nouveau concept d'aménagement portuaire répondant à la demande croissante d'emplacements pour les plaisanciers. Il s'agit du procédé breveté BlueRing $^{\circledR}$ (BLUERING, site web) intégrant une dimension verticale dans les infrastructures portuaires grâce à l'utilisation de la technique des "parois moulées » dites «auto-stables» (SOLETANCHE-BACHY, site web). Cette technique est mondialement éprouvée car elle permet la construction d'ouvrages souterrains profonds et étanches à l'eau, tels que des parkings urbains, des puits d'accès pour tunneliers ou des formes de radoub.

Les atouts de BlueRing $^{\circledR}$ (figure 1) sont multiples : impact visuel favorable, occupation optimale des surfaces, insertion aisée en site urbain, création d'un espace souterrain à usages variés (port à sec, parking, ...), coût réduit lié à la lutte contre l'envasement, possibilité d'occuper des sols ayant des qualités géotechniques médiocres.

$$
\text { DOI: } 10.5150 / \mathrm{cmcm} .2009 .015
$$


Cette note a pour objectif la modélisation de l'agitation dans un bassin portuaire de forme annulaire, connecté avec l'extérieur par un canal d'accès qui est aligné de telle sorte que l'onde incidente suit une radiale vers le centre du port. Cette modélisation doit permettre de définir les conditions nécessaires pour que les vagues de faible hauteur ne s'amplifient pas dans les bassins construits selon le concept BlueRing ${ }^{\circledR}$, sachant que ce type d'aménagement est prévu pour des sites abrités de la houle océanique.

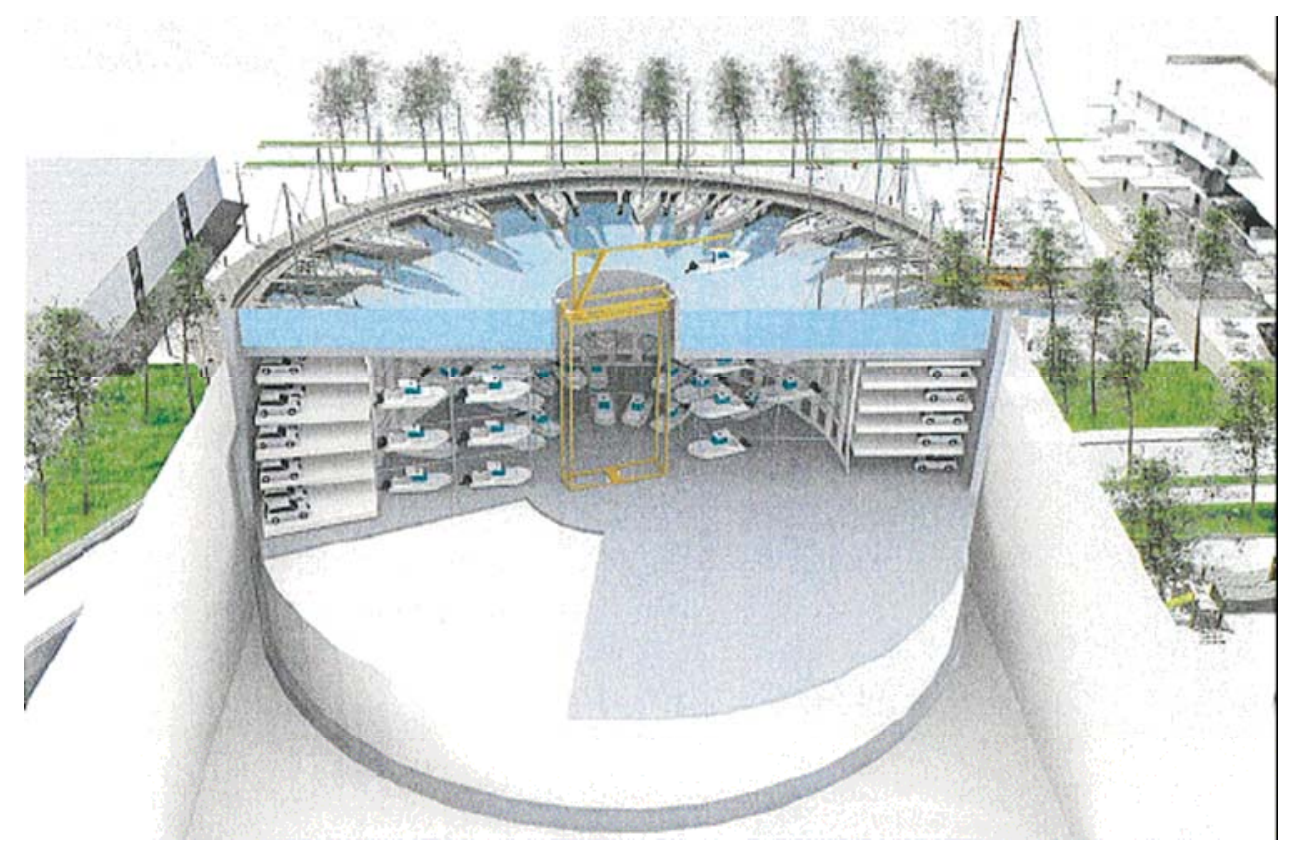

Figure 1. Illustration d'un port de plaisance avec parking souterrain implanté en site urbain suivant le concept BlueRing ${ }^{\circledR}$.

\section{Méthodes}

\subsection{Théorie}

Dans cette note il est admis que les vitesses induites par les vagues dérivent d'un potentiel complexe qui satisfait les conditions aux limites propres à un fond imperméable et à une surface libre isobare. Ce potentiel $\Phi$ s'écrit comme suit :

$\Phi=-\frac{i g a_{i}}{\omega} \phi(r, \theta) \frac{\cosh [k(z+d)]}{\cosh [k d]} \exp (-i \omega t)$

où $\phi(r, \theta)$ désigne une fonction bidimensionnelle complexe qui doit être déterminée cas par cas, $r$ et $\theta$ sont les coordonnées polaires du plan horizontal, $z$ la coordonnée verticale (positive vers le haut et ayant pour origine le niveau de repos), $t$ le temps, $i$ la racine carrée de -1, g l'accélération de la pesanteur, $a_{i}$ l'amplitude de l'onde incidente de référence qui se dirige vers le centre du port par le canal d'accès, $d$ la profondeur, $k$ le nombre d'onde et $\omega$ la fréquence angulaire. Les grandeurs $k$ et $\omega$ sont liées par :

$\omega^{2}=g k \tanh (k d)$ 
La fonction $\phi(r, \theta)$ caractérise l'amplitude des oscillations et la phase dans chaque point du domaine examiné. Dans ce domaine, si $k$ est constant, cette fonction est régie par l'équation de Helmholtz qui en coordonnées polaires s'écrit comme suit :

$\frac{1}{r} \frac{\partial}{\partial r}\left(r \frac{\partial \phi}{\partial r}\right)+\frac{1}{r^{2}} \frac{\partial^{2} \phi}{\partial \theta^{2}}+k^{2} \phi=0$

La solution de cette équation doit satisfaire à des conditions aux limites appropriées, tenant compte de la houle incidente et des éventuelles réflexions au niveau des frontières délimitant le domaine étudié (ISAACSON \& QU, 1990 ; SANCHEZ, 1998).

\subsection{Discrétisation du problème}

La méthode des différences finies est utilisée pour résoudre l'équation de Helmholtz. Le maillage comporte $\mathrm{M}=30$ cercles concentriques équidistants et $\mathrm{N}=120$ radiales espacées avec un pas angulaire $\Delta \theta=2 \pi / \mathrm{N}$ rad.

\subsection{Définition d'un paramètre pour quantifier l'amplification des vagues}

Un coefficient d'amplification moyen quadratique $Z_{r m s}$ calculé par intégration sur la surface du bassin est utilisé pour décrire les résultats relatifs à l'agitation portuaire. Selon les paramètres du problème, trois résultats sont possibles :

a) $Z_{r m s}<1$, en moyenne, les vagues incidentes s'amortissent dans le bassin,

b) $Z_{r m s}=1$, en moyenne, les vagues incidentes restent constantes dans le bassin,

c) $Z_{r m s}>1$, en moyenne, les vagues incidentes s'amplifient dans le bassin.

\section{Résultats}

La figure 2(a) montre pour un cas étudié, la variation de $Z_{r m s}$ en fonction d'une longueur d'onde réduite. La courbe continue en rouge correspond à une paroi externe complètement réfléchissante, en pointillés sont tracés les résultats relatifs à des parois externes partiellement réfléchissantes qui sont caractérisées par un coefficient de réflexion $K r$, et enfin, la courbe continue en noir correspond à une paroi externe complètement absorbante $(K r=0)$.

La figure 2(b) montre pour un cas étudié, des résultats relatifs à un bassin comportant une paroi radiale située derrière le puits central par rapport à l'accès. L'ensemble de ces résultats est lié à une paroi externe complètement réfléchissante $(K r=1)$. La courbe continue en rouge correspond à une paroi radiale complètement réfléchissante $(K r=1)$, en pointillés sont tracés les résultats relatifs à des parois radiales partiellement réfléchissantes, et la courbe continue en noir correspond à une paroi radiale complètement absorbante $(K r=0)$.

D'autres cas complémentaires ont été étudiés (résultats non montrés) couvrant des diamètres du bassin variant de 50 à $200 \mathrm{~m}$, des diamètres du puits compris entre 5 et $20 \mathrm{~m}$, des longueurs d'onde réduites (par le rayon du bassin) variant entre 0.2 et 2.4. 

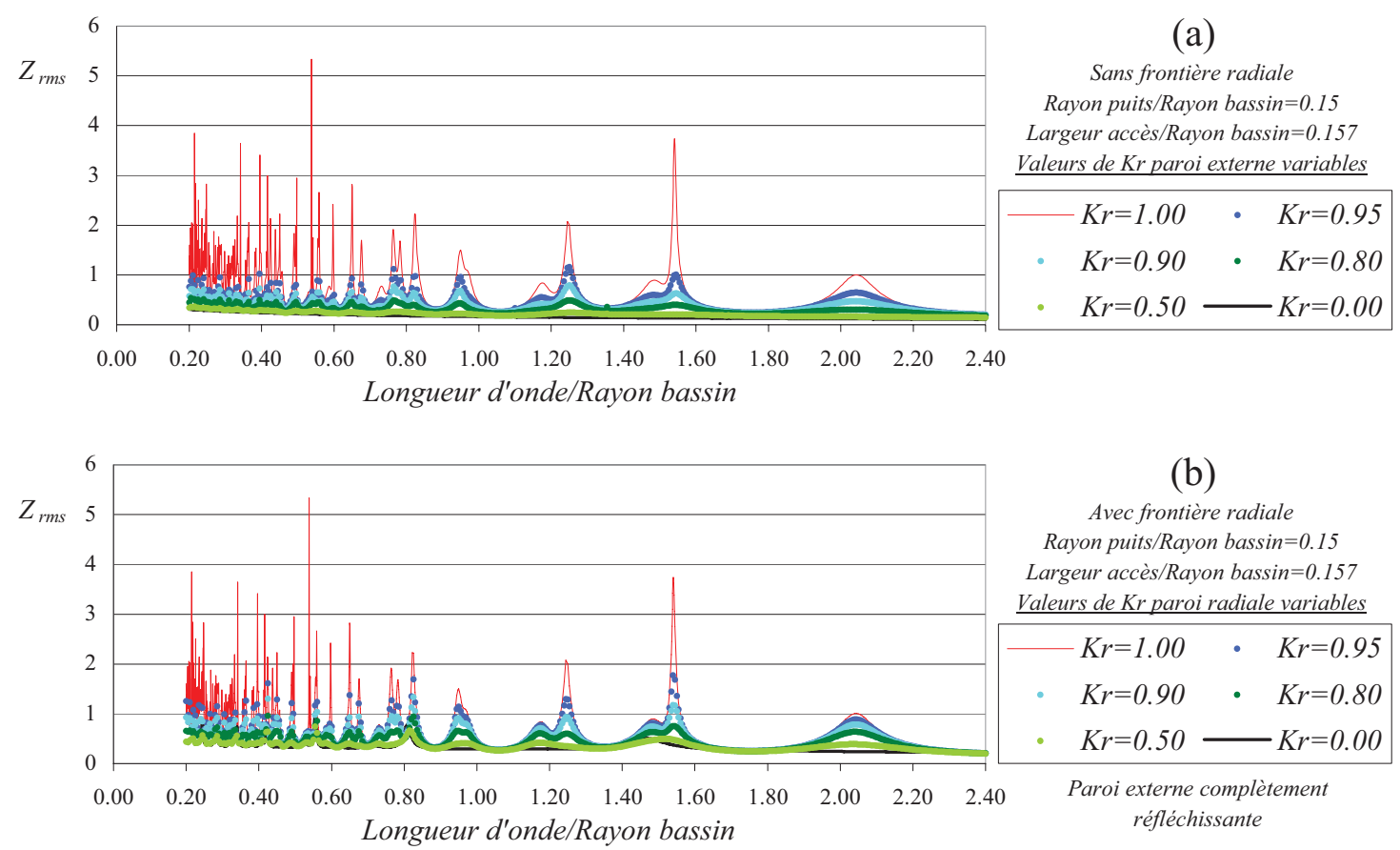

Figure 2. Variation de $Z_{r m s}$ en fonction d'une longueur d'onde réduite.

\section{Discussion et conclusions}

Dans les deux cas correspondant aux résultats montrés sur les figures 2(a) et 2(b), et sur tous les cas complémentaires étudiés, il est observé que si toutes les parois sont complètement réfléchissantes, les vagues incidentes peuvent s'amplifier dans le bassin pour certaines longueurs d'onde.

Pour obtenir un amortissement de la houle, quelles que soient la longueur d'onde et la dimension du port (dans la limite des cas étudiés) deux solutions sont possibles :

a) la mise en place d'une paroi externe caractérisée par une valeur de $K r \leq 0.9$.

b) la mise en place d'une paroi radiale derrière le puits central par rapport à l'accès, caractérisée par $K r \leq 0.8$.

Enfin, il doit être noté que l'agitation portuaire ne pose aucun problème significatif quand la hauteur de la houle incidente est petite, de l'ordre de $20 \mathrm{~cm}$, même si toutes les frontières du port sont complètement réfléchissantes.

\section{Références}

BLUERING (site web). http://www.bluering.fr/

ISAACSON M., QU S. (1990). Waves in a harbour with partially reflecting boundaries. Coastal Engineering, Vol. 14, $\mathrm{n}^{\circ} 3$, pp 193-214.

SANCHEZ M. (1998). Agitation portuaire : modélisation et mesure. V ${ }^{\text {èmes }}$ Journées Nationales Génie Civil - Génie Côtier, Toulon, pp 55-64. [URL http://www.paralia.fr/Files/05_07_10p_sanchez.pdf]

SOLETANCHE-BACHY (site web).

http://www.soletanche-bachy.com/SBF/referencesb.nsf/Tech/parois.moulees.en.beton.arme 